\title{
Deregulated expression of selected histone methylases and demethylases in prostate carcinoma
}

\author{
Filipa Quintela Vieira',2, Pedro Costa-Pinheiro', João Ramalho-Carvalho', \\ Andreia Pereira', Francisco Duarte Menezes ${ }^{3}$, Luís Antunes ${ }^{4}$, Isa Carneiro, ${ }^{1,3}$, \\ Jorge Oliveira $^{5}$, Rui Henrique ${ }^{1,3,6, *}$ and Carmen Jerónimo ${ }^{1,6, *}$ \\ ${ }^{1}$ Cancer Epigenetics Group, Portuguese Oncology Institute, Research Center, Rua Dr António \\ Bernardino Almeida, 4200-072 Porto, Portugal \\ ${ }^{2}$ School of Allied Health Sciences (ESTSP), Polytechnic of Porto, Portugal \\ Departments of ${ }^{3}$ Pathology ${ }^{4}$ Epidemiology ${ }^{5}$ Urology, Portuguese Oncology Institute, Porto, Portugal \\ ${ }^{6}$ Department of Pathology and Molecular Immunology, Institute of Biomedical Sciences Abel Salazar (ICBAS), \\ University of Porto, Porto, Portugal \\ *(R Henrique and C Jerónimo are joint senior authors)
}

\begin{abstract}
Prostate cancer ( $\mathrm{PCa}$ ), a leading cause of cancer-related morbidity and mortality, arises through the acquisition of genetic and epigenetic alterations. Deregulation of histone methyltransferases (HMTs) or demethylases (HDMs) has been associated with PCa development and progression. However, the precise influence of altered HMTs or HDMs expression and respective histone marks in PCa onset and progression remains largely unknown. To clarify the role of HMTs and HDMs in prostate carcinogenesis, expression levels of 37 HMTs and 20 HDMs were assessed in normal prostate and PCa tissue samples by RT-qPCR. SMYD3, SUV39H2, PRMT6, KDM5A, and KDM6A were upregulated, whereas KMT2A-E (MLL1-5) and KDM4B were downregulated in PCa, compared with normal prostate tissues. Remarkably, PRMT6 was the histone modifier that best discriminated normal from tumorous tissue samples. Interestingly, EZH2 and SMYD3 expression levels significantly correlated with less differentiated and more aggressive tumors. Remarkably, SMYD3 expression levels were of independent prognostic value for the prediction of disease-specific survival of PCa patients with clinically localized disease submitted to radical prostatectomy. We concluded that expression profiling of HMTs and HDMs, especially SMYD3, might be of clinical usefulness

for the assessment of PCa patients and assist in pre-therapeutic decision-making.
\end{abstract}

Correspondence should be addressed to $C$ Jerónimo Email carmenjeronimo@ipoporto. min-saude.pt or cljeronimo@icbas.up.pt
Endocrine-Related Cancer (2014) 21, 51-61

\section{Introduction}

Prostate cancer (PCa) is the most frequently diagnosed cancer in men from developed countries and a leading cause of cancer-related morbidity and mortality worldwide (Jemal et al. 2011, Siegel et al. 2012). At its earliest stages, PCa is frequently asymptomatic, fostering the use of biomarkers, such as serum prostate-specific antigen (PSA), for screening

Key Words
- prostate
- neoplasia
- molecular biology
- biomarker
- microarray


other prognostic factors used for therapeutic decision (e.g., clinical stage and pre-therapeutic serum PSA levels) are rather imperfect in predicting disease progression (Lapointe et al. 2004, Duffy 2011). Consequently, there is a significant degree of uncertainty concerning the threat that a PCa poses to an individual patient, entailing overtreatment (Moyer 2012).

The role of epigenetic modifications in cancer initiation and progression has been emphasized (Hirst \& Marra 2009). In addition to aberrant DNA methylation, alterations in chromatin modification patterns, due to histones post-translational modifications (PTMs), were implicated in carcinogenesis and have emerged as potential key regulators of cancer-related pathways (Miremadi et al. 2007). Importantly, PTMs may be changed in cancer cells due to altered expression or activity of key chromatin-modifying enzymes (Miremadi et al. 2007).

Histone methylation, carried out by histone methyltransferases (HMTs), requires different families of enzymes depending on the residue (lysine HMTs (KMT) methylate lysine residues, whereas protein arginine methyltransferase (PRMT) methylate arginines) and might positively or negatively regulate gene transcription. Although lysine residues might be modified into mono-, di-, or trimethyl states, arginine can only be modified to mono- or dimethyl states (symmetric or asymmetrically) (Brame et al. 2004). Different degrees of methylation may be, thus, associated with distinct chromatin regions or transcriptional states (e.g., trimethylation of lysine 9 of histone $\mathrm{H} 3$ is associated with pericentromeric heterochromatin and transcriptional repression, whereas its dimethylation is linked to repressed genes in euchromatin (Lee et al. 2006)). Recently, the reversibility of histone methylation has been established through the discovery of histone lysine and arginine demethylases (HDMs), uncovering a new level of histone plasticity (Shi et al. 2004, Chang et al. 2007).

Altered HMTs expression levels have been found in PCa, most notably enhancer of zeste homolog 2 (EZH2), a lysine methyltransferase, which is increased in metastatic PCa, marking aggressive disease (Seligson et al. 2005, Karanikolas et al. 2010). Specific relationships between histone marks and tumor grade or recurrence (particularly methylation of H3K4 and H3K27) have been reported (Seligson et al. 2009, Bianco-Miotto et al. 2010) and deregulation of some lysine HMTs - KMT2B, KMT2C, NSD1, EZH2 or SMYD3 - in PCa tissues has been also demonstrated (Ke et al. 2009, Bianco-Miotto et al. 2010). However, the validity of most studies is limited due to inappropriate tissue sampling and/or to the reduced number of samples tested.

Because deregulation of HMTs and HDMs affects post-translational control of cellular proteins involved in cancer-relevant signaling networks, a better understanding of their function might lead to the identification of more accurate markers that might be useful to discriminate patients benefiting from a more aggressive treatment from those that might be spared unnecessary and potentially harmful interventions. Therefore, we sought to identify HMTs and HDMs displaying altered expression levels, in a relatively large series of PCa patients submitted to radical prostatectomy, and further test their clinical usefulness for the prediction of disease progression.

\section{Materials and methods}

\section{Patients and tissue collection}

Primary tumors from 160 patients with clinically localized prostate adenocarcinoma, consecutively diagnosed, and primarily treated with radical prostatectomy at the Portuguese Oncology Institute, Porto, Portugal, were prospectively collected. For control purposes, non-neoplastic prostate tissue samples were obtained from the peripheral zone of 15 prostates not harboring $\mathrm{PCa}$ collected from cystoprostatectomy specimens of patients with bladder cancer (normal prostate tissue (NPT)). All tissue specimens were promptly frozen immediately after surgery, following informed consent. Five-micron thick sections were cut and stained for the identification of the areas of PCa (i.e., the index or dominant tumor) and normal tissue. Then, the tissue block was trimmed to maximize the yield of target cells ( $>70 \%$ of target cells). Subsequently, an average of fifty $12-\mu \mathrm{m}$ thick sections was cut and every fifth section was stained to ensure a uniform percentage of target cells and to exclude contamination from neoplastic cells in normal tissue samples. Histological slides from formalin-fixed, paraffin embedded tissue fragments were routinely obtained from the same surgical specimens and assessed for Gleason score (GS; Gleason \& Mellinger 1974) and TNM stage (Hermanek et al. 1997). Relevant clinical data were collected from the clinical records. These studies were approved by the institutional review board (Comissão de Ética para a Saúde-(IRB-CES-IPOFG-EPE 019/08)) of Portuguese Oncology Institute, Porto, Portugal.

\section{RNA isolation}

All tissue samples were suspended in TRIzol reagent (Invitrogen) and, after addition of chloroform to the lysed cells, total RNA was purified from the aqueous phase of TRIzol extract using the PureLink RNA Mini Kit (Invitrogen)

Published by Bioscientifica Ltd. 
following manufacturer recommendations. The concentration, purity, and integrity of RNA samples were determined on a Nanodrop ND-1000 (ThermoScientific, Wilmington, DE, USA) and agarose-gel electrophoresis.

\section{Screening of HMTs and HDMs}

Five NPTs and ten independent PCa samples were chosen to encompass the full spectrum of prostate carcinomas in this series considering the GS and pathological stage (Table 1). After treatment with DNase Turbo DNA-free (Ambion, Austin, TX, USA), a total of $1 \mu \mathrm{g}$ total RNA was reverse transcribed using the High Capacity cDNA RT kit (Applied Biosystems) according to the manufacturer's instructions. TaqMan Array 96-Well Plates were designed in order to evaluate expression levels of 37 HMTs and 20 HDMs. RT-qPCR protocol was performed on an ABI- 7500 RealTime PCR system (Applied Biosystems) according to manufacturer's instructions and each sample was run in triplicate.

The amount of mRNAs of the genes studied was normalized to that of the GUSB reference gene and the median value of NPTs and PCa samples was chosen to calculate fold-difference in gene expression between groups, using the comparative $\mathrm{Ct}$ method. Genes with a logarithmized fold change above 0.5 or below -0.5 were further considered. The expression of KDM6A was also included because it has been previously reported as deregulated in several tumor models (van Haaften et al. 2009), and analysis was extend to all members of the KMT2 family.

\section{Validation of selected enzymes}

After gene selection, mRNA levels were confirmed in a large and independent group of 150 PCa tissues and
15 NPTs. A total of $300 \mathrm{ng}$ was reverse transcribed and amplified using TransPlex Whole Transcriptome Amplification Kit (Sigma-Aldrich) with subsequent purification using QIAquick PCR Purification Kit (Qiagen), according to manufacturer's instructions. HMTs or HDMs mRNA levels were evaluated using TaqMan Gene Expression Assays (Applied Biosystems, Supplementary Table 1, see section on supplementary data given at the end of this article) and the most suitable endogenous control assays for the analysis of prostate tissues (de Kok et al. 2005), GUSB, and TFRC were also analyzed. To determine the relative expression levels in each sample, the values of the target gene were normalized using the median of the two internal reference genes to obtain a ratio (HMT or HDM/Mean of TFRC and GUSB). Each plate included multiple non-template controls and serial dilutions of a cDNA from human prostate RNA (Ambion, Invitrogen) to construct a standard curve for each plate. All experiments were run in triplicate.

\section{Statistical analysis}

For statistical analysis purposes, PCa samples were divided into two- or three-grade categories for GS (GS $\leq 6$ and GS $\geq 7$ ) and pathological stage (pT2, pT3a, and pT3b) respectively. The Shapiro-Wilk's $W$-test allowed for the examination of the appropriateness of a normal distribution assumption for each of the parameters (data not shown). Then, the median and range of the mRNA expression levels for each group of samples were determined and analyzed using Mann-Whitney $U$ test. A receiver operator characteristics (ROC) curve was constructed by plotting the true positive rate (sensitivity) against the false positive rate (1-specificity) and the area

Table 1 Clinical and pathological features of patients included in the testing set and in the validation series

\begin{tabular}{|c|c|c|c|c|}
\hline & PCa & Normal & PCa & Normal \\
\hline Number of patients, $n$ & 10 & 5 & 150 & 15 \\
\hline $\begin{array}{l}\text { Age (years), median } \\
\text { (range) }\end{array}$ & $59(53-71)$ & $61(49-66)$ & $64(49-75)$ & $64(45-80)$ \\
\hline $\begin{array}{l}\text { PSA levels (ng/ml), } \\
\text { median (range) }\end{array}$ & $12.3(3.5-19.9)$ & NA & $8.2(2.9-23.0)$ & NA \\
\hline Pathological stage, $n(\%)$ & & NA & & NA \\
\hline pT2 & $4(40.0)$ & & $89(59.3)$ & \\
\hline рT3a & $2(20.0)$ & & $50(33.3)$ & \\
\hline pT3b & $4(40.0)$ & & $11(7.3)$ & \\
\hline Gleason score, $n(\%)$ & & NA & & NA \\
\hline$<7$ & $3(30.0)$ & & $57(38.7)$ & \\
\hline$\geq 7$ & $7(70.0)$ & & $93(62.0)$ & \\
\hline
\end{tabular}

PCa, prostate cancer; NA, not applicable.

http://erc.endocrinology-journals.org DOI: $10.1530 /$ ERC-13-0375
(C) 2014 Society for Endocrinology Printed in Great Britain
Published by Bioscientifica Ltd 
under the curve (AUC) was calculated to assess diagnostic performance. Possible correlations between the expression levels and GS or pathological stage were assessed by the Kruskal-Wallis one-way ANOVA, followed by Mann-Whitney $U$ test when appropriate. For multiple comparisons, the Bonferroni method was used to adjust $P$ values. Spearman nonparametric correlation tests were additionally carried out to ascertain correlations between age, PSA levels, and HMTs or HDMs expression levels. The prognostic significance of available clinical variables (pathological stage, GS, age, and serum PSA levels) was assessed by constructing disease-specific and disease-free survival (DFS) curves using the Kaplan-Meier method with log-rank test (univariate test). A Cox-regression model comprising the four variables (multivariate test) was also constructed. DFS was calculated from the date of the radical prostatectomy to the date of biochemical relapse, or date of last follow-up, or death if relapse-free. For the purposes of survival analyses, all cases were coded based on the expression levels of each enzyme using the percentile 75 value as empirical threshold. Cases were also subdivided according to serum PSA levels (below and above median values) and age (above 60, between 60 and 70, and above 70). Statistical analysis was performed using SPSS for Windows, version 20.0 (SPSS) and the level of significance was set to $P<0.05$. Graphs were built using GraphPad Prism 5.0 software for Windows (GraphPad Software, Inc., La Jolla, CA, USA).

\section{Results}

\section{Evaluation of HMTs and HDMs expression levels}

Expression levels of 37 HMTs and 20 HDMs were assessed in ten PCa and five normal prostate samples (relevant clinical and pathological data are depicted in Table 1). Most enzymes were downregulated in PCa compared with NPT (Fig. 1). Based on fold-variation, lysine HMTs SUV39H2, SMYD3, KMT2A-E, and EZH2 (the latter used as positive control according to the literature); argine HMT PRMT6; and HDMs KDM4B, KDM6A, KDM5A, and KDM3B were selected for validation (Supplementary Table 2, see section on supplementary data given at the end of this article). This was performed using RT-qPCR in a larger and independent series comprising 150 PCa samples and 15 NPTs (relevant clinical and histopathological data are displayed in Table 1). Statistically significant differences between NPT and PCa tissue samples were found for all candidate genes, except KDM3B (Table 2). As expected, higher EZH2 expression levels were observed in PCa compared with NPT and the same trend was verified for SMYD3, SUV39H2, PRMT6, KDM5A, and KDM6A (Fig. 2 and Table 2). Contrarily, all selected members of KMT2 family and $K D M 4 B$ were downregulated in PCa samples compared with NPT (Fig. 3 and Table 2). Interestingly, significant positive correlations between several members of KMT2 family were found in PCa samples (Table 3). To reinforce the oncogenic role of altered enzyme expression, transcript levels (categorized according to percentile 75) were tested as PCa biomarkers in tissue samples. Remarkably, PRMT6 performed best in sensitivity (90.0\%) and specificity (73.3\%) for discriminating PCa from NPT, and ROC curve analysis showed an AUC of 0.923 (95\% CI 0.870-0.977, $P<0.001$ ) (Fig. 4). No significant differences in age between PCa patients and normal tissue donors were apparent. Statistically significant associations between expression levels of SMYD3 $(P=0.044)$ or KMT2A $(P=0.041)$ and pathological stage were disclosed (higher levels in pT3b cases for both genes, Fig. 5A and B). When the patient cohort was stratified according to GS, increased levels of EZH2 $(P=0.048)$ and KMT2C $(P=0.018)$ were associated with less differentiated tumors (Fig. $5 \mathrm{C}$ and D). No statistically significant associations were found between gene expression levels and patients' age or PSA levels.

\section{Survival analysis}

The median follow-up period of this series of PCa patients was 105 months (range: 3-145 months). At the time of the last follow-up, five patients (3.3\%) had died from PCa and 45 of 136 (33\%) presented biochemical recurrence. In 14 patients, serum PSA levels $>0.2 \mathrm{ng} / \mathrm{ml}$ persisted following surgery and these were not further considered for DFS analysis. Disease-specific survival curves using established clinical variables or expression levels of selected genes did not display prognostic value within the available follow-up time. However, DFS analysis showed that tumors with higher transcript levels of EZH2 $(P=0.001)$ or SMYD3 $(P=0.010)$ were significantly associated with a shorter time to relapse, in a univariate analysis (Fig. 6). Higher GS $(P<0.001)$, advanced pathological stage (pT3a $P=0.016$ and pT3b $P=0.002$ ), and higher PSA levels $(P=0.029)$ were also associated with shorter DFS, whereas age was not of prognostic value within the available followup time. In multivariate analysis, higher GS, stage pT3b, and high SMYD3 expression levels retained statistical significance $(P=0.001, \quad P=0.027, \quad$ and $P=0.025$ respectively) and were capable of predicting prognosis independently, whereas EZH2 expression, PSA, and

Published by Bioscientifica Ltd. 

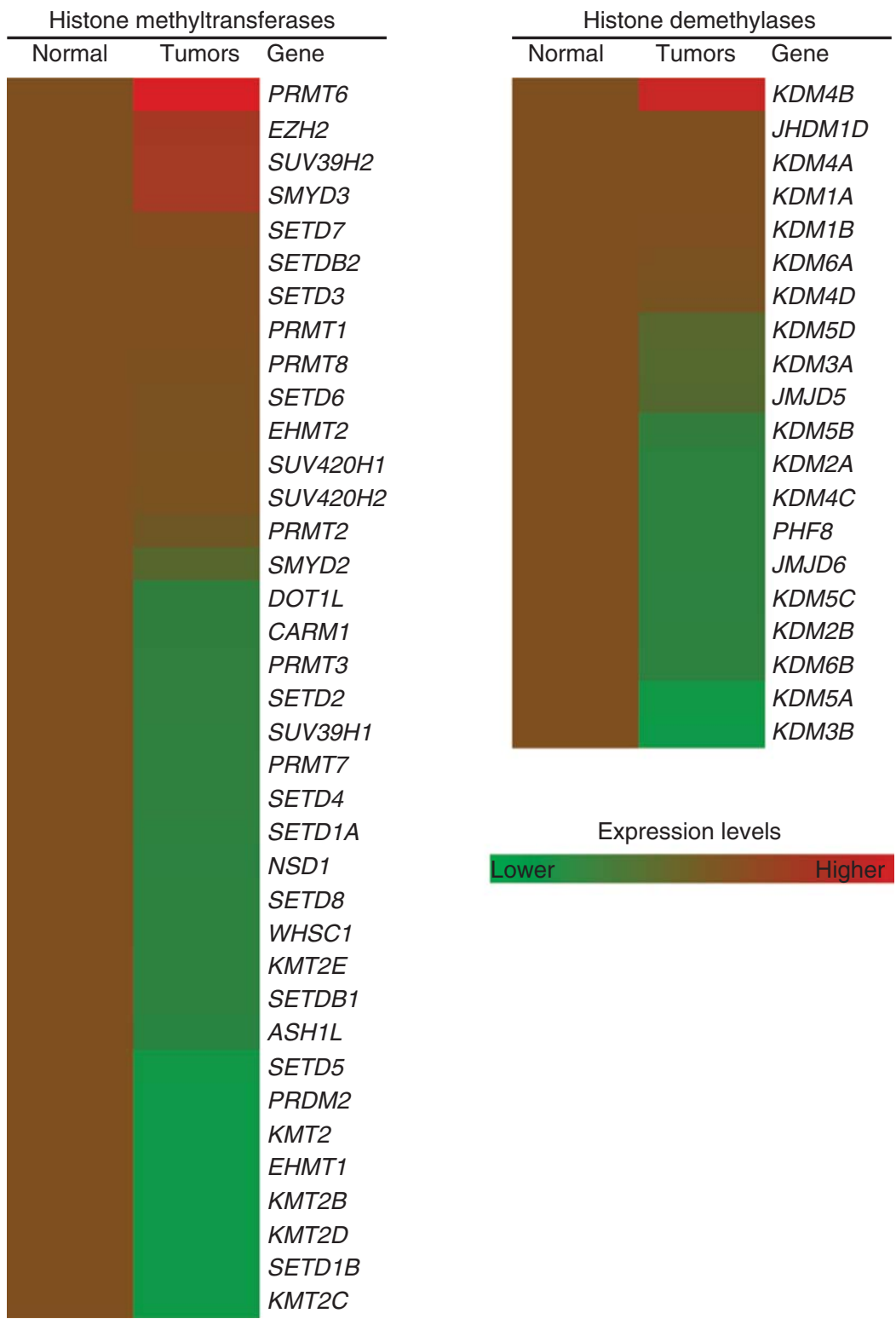

\section{Figure 1}

Expression levels of 37 HMTs and 20 HDMs in normal and PCa tissues. Gene expression of five normal prostate tissues and ten PCa calculated using comparative $\mathrm{Ct}$ method. The results presented correspond to median value of each group.

pathological stage pT3a did not show independent prognostic value, in this dataset (Table 4).

\section{Discussion}

Deregulation of histone PTM patterns has been associated with PCa development and progression (Seligson et al. 2005, Ke et al. 2009, Bianco-Miotto et al. 2010). Because these modifications might be due to altered expression or activity of key chromatin-modifying enzymes (Miremadi et al. 2007), we attempted to globally characterize alterations in expression affecting HMTs and HDMs in PCa tissues and determine whether those might be of clinical and pathological relevance.

Overall, 37 HMTs and 20 HDMs expression levels were assessed, comprising most of the relevant members of each class. Owing to the relatively large number of genes tested, this panel was initially tested in a small series of tissue samples. This might underestimate the frequency and magnitude of changes in gene expression, but it allows for

Published by Bioscientifica Ltd. 
Table 2 Distribution of selected HMTs and HDMs expression levels in normal and PCa tissue samples

\begin{tabular}{l}
\hline Gene \\
\hline EZH2 \\
KMT2A \\
KMT2B \\
KMT2C \\
KMT2D \\
KMT2E \\
PRMT6 \\
SMYD3 \\
SUV39H2 \\
KDM3B \\
KDM4B \\
KDM5A \\
KDM6A
\end{tabular}

\begin{tabular}{l}
\multicolumn{1}{c}{ Normal } \\
\hline $0.77(0.39-1.82)$ \\
$0.69(0.21-2.52)$ \\
$3.23(1.61-6.76)$ \\
$2.37(1.24-4.40)$ \\
$3.91(1.36-10.21)$ \\
$0.73(0.58-3.46)$ \\
$0.16(0.02-0.30)$ \\
$0.90(0.53-1.44)$ \\
$1.07(0.63-2.48)$ \\
$0.26(0.03-0.45)$ \\
$2.24(0.47-7.81)$ \\
$0.21(0.10-0.63)$ \\
$0.33(0.03-0.52)$ \\
\hline
\end{tabular}

\begin{tabular}{c}
\hline Tumor \\
\hline $1.15(0.09-4.85)$ \\
$0.29(0.08-1.30)$ \\
$2.05(0.49-8.07)$ \\
$1.45(0.39-4.06)$ \\
$1.97(0.36-8.66)$ \\
$0.53(0.21-1.55)$ \\
$0.43(0.10-1.77)$ \\
$1.53(0.53-4.50)$ \\
$1.36(0.32-3.43)$ \\
$0.24(0.11-0.78)$ \\
$0.47(0.08-2.23)$ \\
$0.32(0.07-0.77)$ \\
$0.47(0.20-1.58)$ \\
\hline
\end{tabular}

\begin{tabular}{c}
\hline P value, M-W \\
\hline $\mathbf{0 . 0 1 4}$ \\
$<\mathbf{0 . 0 0 1}$ \\
$\mathbf{0 . 0 0 4}$ \\
$<\mathbf{0 . 0 0 1}$ \\
$<\mathbf{0 . 0 0 1}$ \\
$<\mathbf{0 . 0 0 1}$ \\
$<\mathbf{0 . 0 0 1}$ \\
$<\mathbf{0 . 0 0 1}$ \\
$\mathbf{0 . 0 4 4}$ \\
$\mathrm{NS}$ \\
$<\mathbf{0 . 0 0 1}$ \\
$\mathbf{0 . 0 2 6}$ \\
$<\mathbf{0 . 0 0 1}$ \\
\hline
\end{tabular}

\begin{tabular}{c}
\hline AUC \\
\hline 0.692 \\
0.212 \\
0.272 \\
0.232 \\
0.272 \\
0.162 \\
0.923 \\
0.855 \\
0.657 \\
0.495 \\
0.098 \\
0.675 \\
0.813
\end{tabular}

HMT, histone methyltransferase; HDM, histone demethylase; PCa, prostate cancer; M-W, Mann-Whitney U test; AUC, area under the curve; NS, not significant.

the selection of the most significantly altered. Thus, to confirm the initial findings in the arrays, a validation study was performed for the selected genes using RT-qPCR and only three out of 12 genes were not confirmed. Importantly, genes that were previously reported to be overexpressed in PCa, such as EZH2 (Karanikolas et al. 2010), surfaced in the array and were confirmed in the large series of PCa, thus validating our initial approach. Furthermore, EZH2 expression was significantly increased in high GS cases, not associating with pathological stage, confirming previous observations (Laitinen et al. 2008).

Interestingly, some of the most significantly altered genes encode for enzymes that display antagonistic functions. Although this might result in a balance between repressive and active PTMs, it must be recalled that the effect in gene expression will depend on the specific genomic locations and how tumor suppressor genes or oncogenes are differentially affected (Hake et al. 2004,
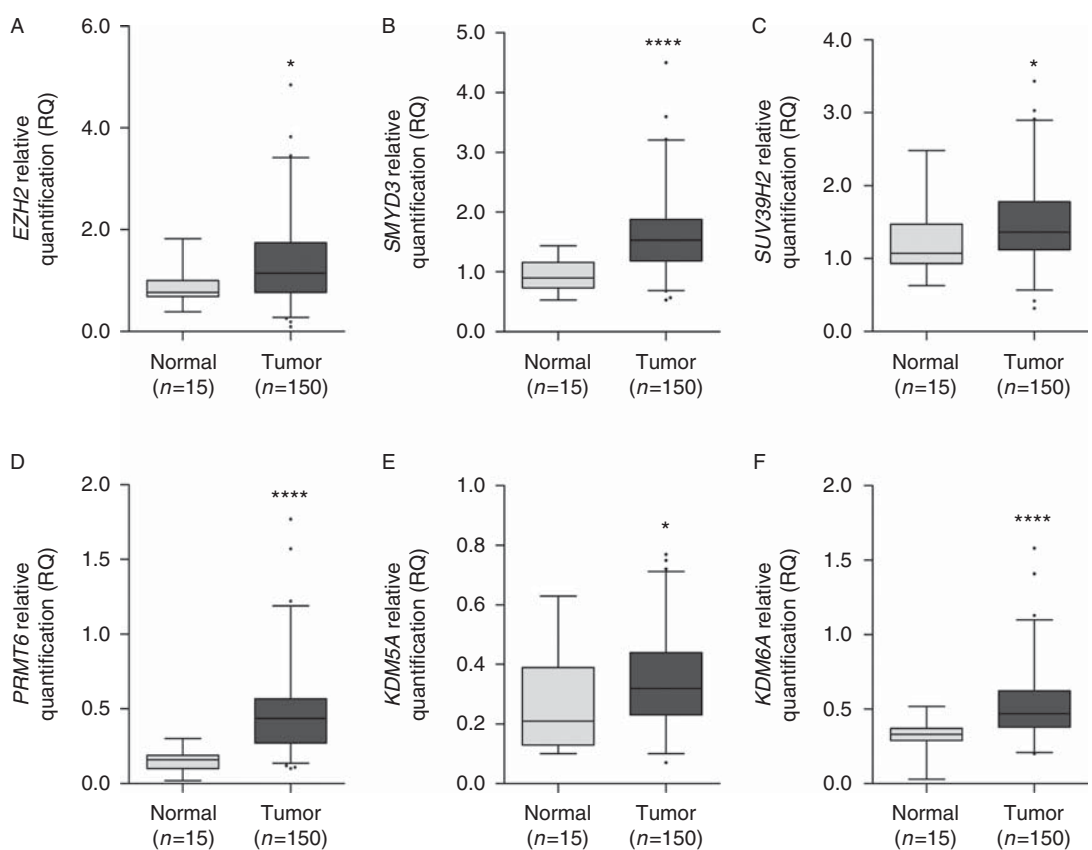

\section{Figure 2}

Identification of HMTs and HDMs overexpressed in PCa. Relative quantification of EZH2 (A), SMYD3 (B), SUV39H2 (C), PRMT6 (D), KDM5A (E), and KDM6A $(\mathrm{F})$, displaying higher expression levels in PCa compared with normal prostate tissues $(* \star * \star P<0.0001 ; * P<0.05)$.

http://erc.endocrinology-journals.org DOI: 10.1530/ERC-13-0375
(C) 2014 Society for Endocrinology Printed in Great Britain
Published by Bioscientifica Ltd 

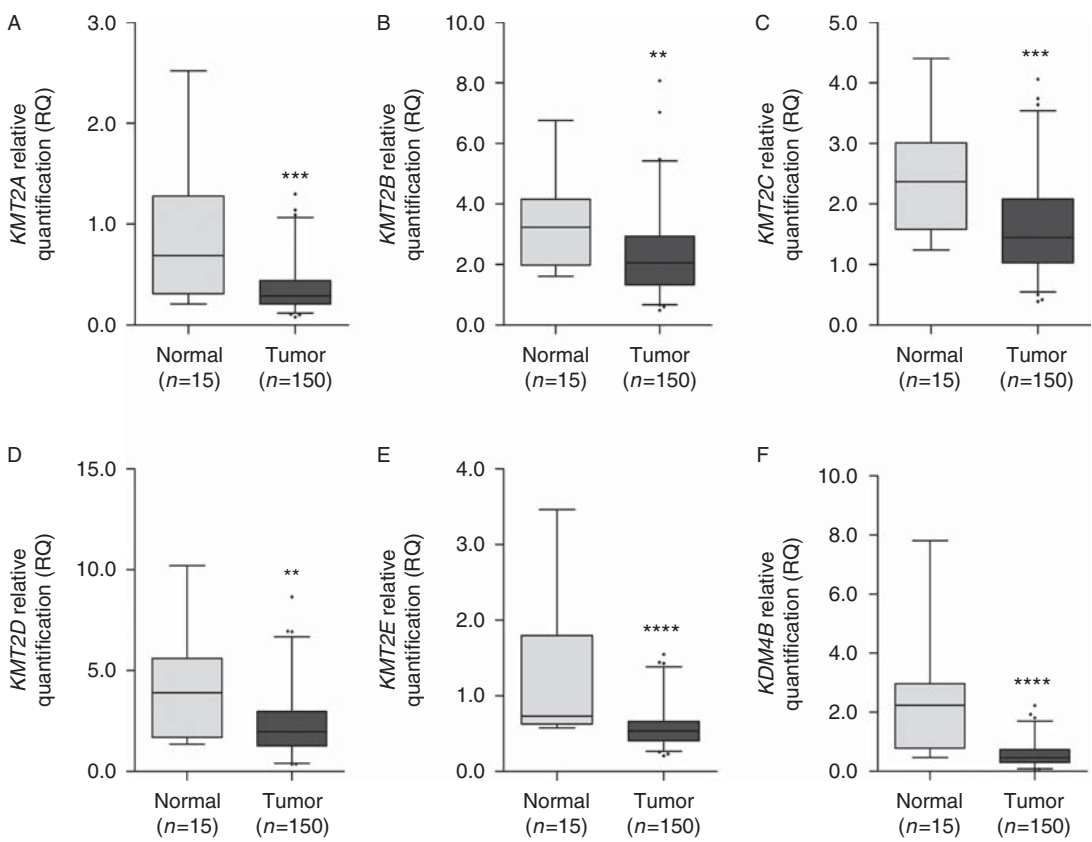

Figure 3

Identification of HMTs and HDMs downregulated in PCa. Relative quantification of KMT2A (A), KMT2B (B), KMT2C (C), KMT2D (D), KMT2E (E), and $K D M 4 B$ (F) depicted lower levels in PCa compared with normal prostate tissues $(* * * * P<0.0001 ; * * * P<0.001 ; * * P<0.01)$.

Chi et al. 2010). Contrarily, concerning the enzymes that have overlapping functions, the same trend was not apparent. This is most likely due to function redundancy, so that oncogenesis is already facilitated through the alteration of a single enzyme responsible for a specific PTM (Fullgrabe et al. 2011).

We found that H3K4 methyltransferase SMYD3 was upregulated in $\mathrm{PCa}$, paralleling previous observations in colorectal, hepatocellular, and breast carcinomas (Hamamoto et al. 2004, 2006), whereas KMT2 family members (which accomplish the same PTM) were downregulated. Remarkably, higher SMYD3 transcript levels were associated with locally advanced disease, suggesting an association with more aggressive PCa. Interestingly, SMYD3 overexpression has been linked with enhanced proliferation and loss of differentiation (Hamamoto et al. 2006, Chen et al. 2007, Wang et al. 2008, Zou et al. 2009, Ren et al. 2011) and this may support the association found in PCa. Moreover, SMYD3 also methylates H4K5 and H4K20 and other non-histone proteins, which may also contribute to its oncogenic role (Foreman et al. 2011, Van Aller et al. 2012). We found that KDM5A, encoding an H3K4 demethylase, was also overexpressed in our PCa series. Remarkably, KDM5A has an antagonistic interaction with pRB, and it is also associated with MYC (Rotili \& Mai 2011), a proto-oncogene which is upregulated in PCa. This putative oncogenic activity, already demonstrated in gastric cancer (Blair et al. 2011), is also supported by our findings.

On the other hand, nearly all members of the KMT2 family were globally downregulated in PCa. This family also targets $\mathrm{H} 3 \mathrm{~K} 4$, but its downregulation might not impact in H3K4me3 levels owing to SMYD3 overexpression. KMT2A and KMT2C displayed higher expression levels in PCa cases with higher GS and more advanced stage, although levels remained lower than those of NPTs. KMT2s operate in complexes (Ansari \& Mandal 2010), a feature that may explain the observed correlation between some members of this family. Because not all KMT2 genes are present in the same complexes, a downstream mechanism responsible for their global downregulation in prostate carcinogenesis likely exists. Furthermore,

Table 3 Coefficient of correlation $(r)$ between the expression levels of all members of KMT2 family in PCa tissue samples

\begin{tabular}{|c|c|c|c|c|c|}
\hline & KMT2A & КМТ2В & КMT2C & KMT2D & KMT2E \\
\hline KMT2A & - & 0.773 & 0.814 & 0.600 & 0.560 \\
\hline КМТ2В & - & - & 0.844 & 0.729 & 0.468 \\
\hline KMT2C & - & - & - & 0.650 & 0.464 \\
\hline$K M T 2 D$ & - & - & - & - & 0.458 \\
\hline KMT2E & - & - & - & - & - \\
\hline
\end{tabular}

PCa, prostate cancer; Spearman correlation; $P<0.001$ for all comparisons.

Published by Bioscientifica Ltd 


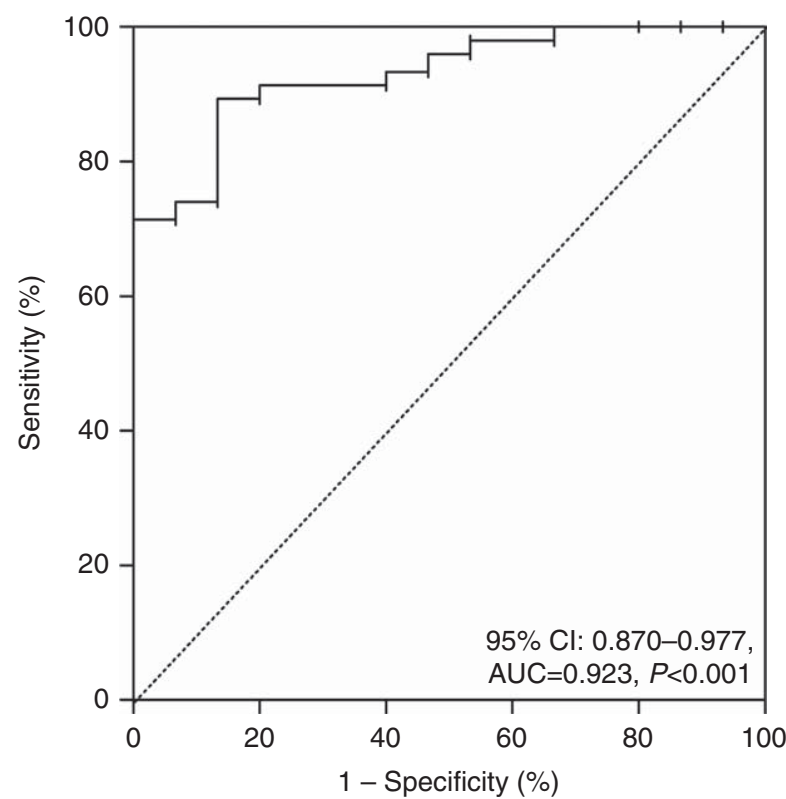

\section{Figure 4}

Performance of PRMT6 expression as biomarker for PCa. Receiver operator characteristic (ROC) curve evaluating the ability of PRMT6 expression levels in discriminating PCa from normal prostate tissues. AUC, area under the curve. a negative crosstalk between methylation of H3R2 by PRMT6 and H3K4 by KMT2 complex was described (Guccione et al. 2007) and increased expression of PRMT6 was identified in our set of PCa. The overexpression of this histone modifier, already reported in bladder and lung cancer, might lead to a decrease in p53 levels, fostering tumorigenesis (Yoshimatsu et al. 2011, Neault et al. 2012). Interestingly, PRMT6 proved to be the HMT that best discriminated PCa from NPTs, further supporting a role for its deregulation in prostate carcinogenesis.

SUV39H2 and KDM4B methylate and demethylate H3K9, respectively. SUV39H2's role in cancer depends on the model: in B-cell lymphomas it acts as a tumor suppressor (Cloos et al. 2008), whereas in breast cancer it is regarded as an oncogene (Franci et al. 2013), as our results suggest for $\mathrm{PCa}$. A positive correlation between KDM4B expression and increased PCa grade has been reported (Coffey et al. 2013), but we were not able to confirm it, probably due to methodological differences. Coffey et al. used samples of benign prostate hyperplasia as controls and this lesion is reported to be potentially linked with PCa arising in the transition zone (Guess 2001). Moreover, their analysis was based on a qualitative evaluation of cytoplasmic immunostaining in a small portion of tissue (Coffey et al. 2013). On the contrary,
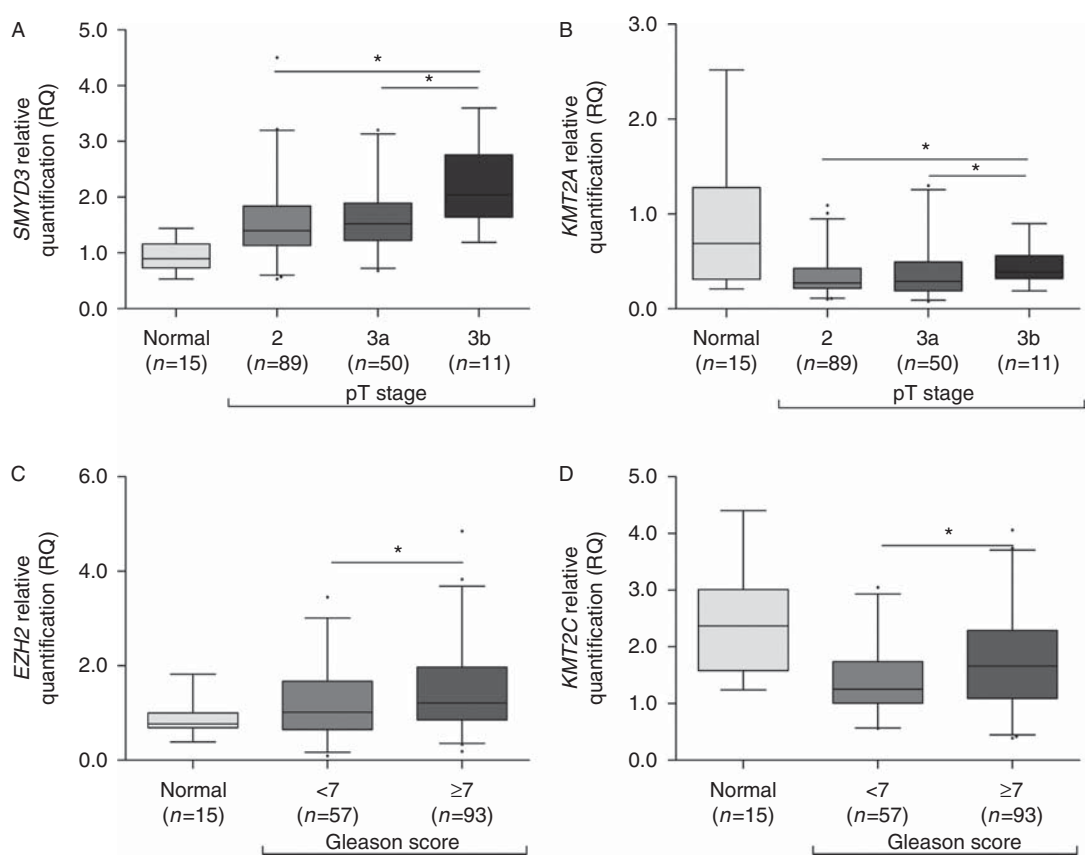

( $\left.{ }^{*} P<0.05\right)$. Distribution of $E Z H 2$ (C) and $K M T 2 C$ (D) expression levels according to Gleason score, displaying higher levels in tumors with Gleason score $\geq 7\left({ }^{*} P<0.05\right)$. SMYD3 (A) and KMT2A (B) expression levels according to pathological stage, showing higher levels in locally advanced disease stage pT3b 

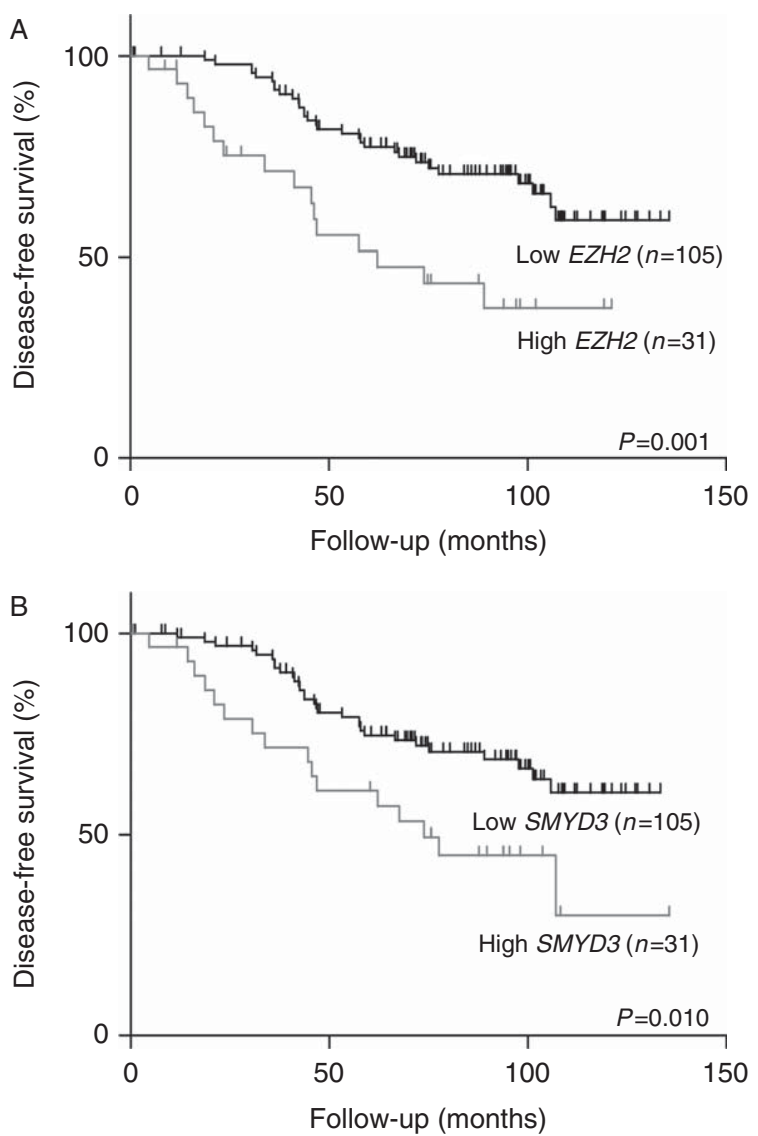

Figure 6

Kaplan-Meier estimated disease-free survival curves for PCa patients. Disease-free survival curves of $136 \mathrm{PCa}$ patients according to expression levels of $E Z H 2$ (A) and SMYD3 (B). The results of RT-qPCR presented were categorized using third quartile (75th percentile) value as the cutoff.

we used morphologically NPT from the peripheral zone, were over $80 \%$ of $\mathrm{PCa}$ originate, and expression was quantitatively assessed at transcript level.

A major goal of our study was to determine the potential clinical usefulness of altered HMTs and HDMs expression in PCa. Only EZH2 and SMYD3 disclosed a significant association with DFS, in univariate analysis. Similar results have been reported for EZH2 expression, although assessed by immunohistochemistry (Varambally et al. 2002, Laitinen et al. 2008, Wolters et al. 2010), and it was found to independently predict PCa recurrence. Although we did not confirm this result for EZH2 at transcript level, a statistical trend was apparent. It should be recalled that our series only incorporates patients with clinically localized PCa, submitted to radical prostatectomy, which represent a subset of the whole spectrum of PCa patients. Because these patients are selected for having clinically organ-confined disease, the corresponding PCas are usually of low and intermediate grade (mostly GS 6 and 7 in the biopsy) and low stage (cT1c and cT2). Thus, it does not comprise the full spectrum of $\mathrm{PCa}$, as clinically advanced and high-grade cases at diagnosis will not be considered (in general) for curative-intent radical prostatectomy. Notwithstanding these limitations, high SMYD3 expression retained prognostic significance in multivariate analysis, confirming its potential clinical usefulness. To more easily translate for routine practice, however, it would be important to determine if immunohistochemical assessment of SMYD3 expression would provide the same result. Though several commercially available antibodies were tested, none provided satisfactory results.

Concerning disease-specific survival, no statistically significant associations were apparent, probably due to relatively short follow-up data. A follow-up period of 15 or 20 years is usually required to detect differences in PCa survival in patients with localized disease submitted to radical prostatectomy (Popiolek et al. 2013). Nonetheless, biochemical recurrence is also an important primary endpoint in many studies. As expected, GS and pathological stage were of prognostic significance in univariate analysis, although only the former and stage pT3b denoted independent prognostic value in multivariate analysis. The fact that stage pT3a did not surfaced as independent prognostic parameter for DFS in multivariate analysis is most likely due to the association between tumor stage and histological grade, as pT3a cancers were mostly of high GS.

Table 4 Cox regression models assessing the potential of clinical and epigenetic variables in the prediction of diseasefree survival for 136 PCa patients

\begin{tabular}{|c|c|c|c|c|}
\hline Gene & Variable & HR & $\begin{array}{c}95 \% \text { Cl for } \\
\text { HR }\end{array}$ & $\begin{array}{l}\text { P value } \\
\text { (CR) }\end{array}$ \\
\hline \multirow[t]{6}{*}{$E Z H 2$} & PSA levels > med & 1.652 & $0.914-2.986$ & 0.096 \\
\hline & Gleason score & 4.206 & $1.820-9.718$ & 0.001 \\
\hline & pT stage $>2$ & & & 0.148 \\
\hline & vs $3 a$ & 1.457 & $0.753-2.819$ & 0.264 \\
\hline & vs $3 b$ & 2.397 & $0.980-5.864$ & 0.055 \\
\hline & $\begin{array}{l}\text { EZH2 expression } \\
\quad>\text { Q75 }\end{array}$ & 1.890 & $0.983-3.637$ & 0.056 \\
\hline \multirow[t]{6}{*}{ SMYD3 } & PSA levels > med & 1.697 & $0.940-3.064$ & 0.079 \\
\hline & Gleason score & 4.259 & $1.817-9.982$ & 0.001 \\
\hline & pT stage 2 & & & 0.086 \\
\hline & vs $3 a$ & 1.476 & $0.755-2.886$ & 0.255 \\
\hline & vs $3 b$ & 2.662 & $1.115-6.356$ & 0.027 \\
\hline & $\begin{array}{l}\text { SMYD3 expression } \\
\quad>\text { Q75 }\end{array}$ & 2.049 & $1.096-3.832$ & 0.025 \\
\hline
\end{tabular}

PCa, prostate cancer; $C R$, Cox regression; HR, hazard ratio; Med, median value; $\mathrm{Q} 75$, quartile 75 value; bold highlights statistical significance $(P<0.05)$.

Published by Bioscientifica Ltd. 
In conclusion, we identified a set of HMTs and HDMs deregulated in PCa that might contribute to the disease development and progression. To the best of our knowledge, this is the first study to demonstrate that HMT SMYD3 expression levels are able to predict disease-specific survival of PCa patients with clinically localized disease, submitted to radical prostatectomy. Therefore, determination of SMYD3 expression levels in prostate biopsies might be able to convey relevant prognostic information in a pretherapeutic setting. Functional studies are mandatory to ascertain the role of SMYD3 in prostate carcinogenesis.

\section{Supplementary data}

This is linked to the online version of the paper at http://dx.doi.org/10.1530/ ERC-13-0375.

\section{Declaration of interest}

The authors declare that there is no conflict of interest that could be perceived as prejudicing the impartiality of the research reported.

\section{Funding}

This study was funded by research grants from Liga Portuguesa Contra o Cancro - Núcleo Regional do Norte, Research Center of Portuguese Oncology Institute - Porto (Cl-IPOP 4-2008) and European Community's Seventh Framework Programme - grant number FP7-HEALTH-F5-2009241783. F Quintela Vieira and J Ramalho-Carvalho are supported by FCTFundação para a Ciência e a Tecnologia grants (SFRH/BD/70564/2010 and

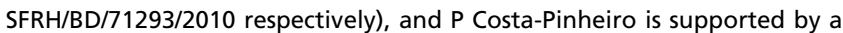
grant from Liga Portuguesa Contra o Cancro - Núcleo Regional do Norte.

\section{Author contribution statement}

F Q Vieira, J Ramalho-Carvalho, R Henrique, and C Jerónimo contributed to experimental design. F Q Vieira, A Pereira, and I Carneiro performed RT-qPCR anaysis. F D Menezes and J Oliveira collect relevant clinical information. $L$ Antunes assisted in statistical analysis. $R$ Henrique performed tumor collection and histopathological classification. F Q Vieira, P Costa-Pinheiro, R Henrique, and C Jerónimo wrote the manuscript with input from co-authors. $\mathrm{R}$ Henrique and $\mathrm{C}$ Jerónimo are joint senior authors.

\section{Acknowledgements}

The authors are grateful to the patients who volunteered to provide samples and also to all the personnel of the Department of Urology, Portuguese Oncology Institute - Porto who kindly collaborated in this study.

\section{References}

Allsbrook WC Jr, Mangold KA, Johnson MH, Lane RB, Lane CG \& Epstein JI 2001 Interobserver reproducibility of Gleason grading of prostatic carcinoma: general pathologist. Human Pathology 32 81-88. (doi:10.1053/hupa.2001.21135)
Ansari KI \& Mandal SS 2010 Mixed lineage leukemia: roles in gene expression, hormone signaling and mRNA processing. FEBS Journal 277 1790-1804. (doi:10.1111/j.1742-4658.2010.07606.x)

Bianco-Miotto T, Chiam K, Buchanan G, Jindal S, Day TK, Thomas M, Pickering MA, O'Loughlin MA, Ryan NK, Raymond WA et al. 2010 Global levels of specific histone modifications and an epigenetic gene signature predict prostate cancer progression and development. Cancer Epidemiology, Biomarkers \& Prevention 19 2611-2622. (doi:10.1158/ 1055-9965.EPI-10-0555)

Blair LP, Cao J, Zou MR, Sayegh J \& Yan Q 2011 Epigenetic regulation by lysine demethylase 5 (KDM5) enzymes in cancer. Cancer 3 1383-1404. (doi:10.3390/cancers3011383)

Brame CJ, Moran MF \& McBroom-Cerajewski LD 2004 A mass spectrometry based method for distinguishing between symmetrically and asymmetrically dimethylated arginine residues. Rapid Communications in Mass Spectrometry 18 877-881. (doi:10.1002/rcm.1421)

Chang B, Chen Y, Zhao Y \& Bruick RK 2007 JMJD6 is a histone arginine demethylase. Science 318 444-447. (doi:10.1126/science.1145801)

Chen LB, Xu JY, Yang Z \& Wang GB 2007 Silencing SMYD3 in hepatoma demethylates RIZI promoter induces apoptosis and inhibits cell proliferation and migration. World Journal of Gastroenterology $\mathbf{1 3}$ 5718-5724.

Chi P, Allis CD \& Wang GG 2010 Covalent histone modificationsmiswritten, misinterpreted and mis-erased in human cancers. Nature Reviews. Cancer 10 457-469. (doi:10.1038/nrc2876)

Cloos PA, Christensen J, Agger K \& Helin K 2008 Erasing the methyl mark: histone demethylases at the center of cellular differentiation and disease. Genes and Development 22 1115-1140. (doi:10.1101/ gad.1652908)

Coffey K, Rogerson L, Ryan-Munden C, Alkharaif D, Stockley J, Heer R, Sahadevan K, O’Neill D, Jones D, Darby S et al. 2013 The lysine demethylase, KDM4B, is a key molecule in androgen receptor signalling and turnover. Nucleic Acids Research 41 4433-4446. (doi:10.1093/nar/gkt106)

Duffy MJ 2011 Prostate-specific antigen: does the current evidence support its use in prostate cancer screening? Annals of Clinical Biochemistry $\mathbf{4 8}$ 310-316. (doi:10.1258/acb.2011.010273)

Foreman KW, Brown M, Park F, Emtage S, Harriss J, Das C, Zhu L, Crew A, Arnold L, Shaaban S et al. 2011 Structural and functional profiling of the human histone methyltransferase SMYD3. PLOS ONE 6 e22290. (doi:10.1371/journal.pone.0022290)

Franci C, Zhou J, Jiang Z, Modrusan Z, Good Z, Jackson E \& Kouros-Mehr H 2013 Biomarkers of residual disease, disseminated tumor cells, and metastases in the MMTV-PyMT breast cancer model. PLOS ONE $\mathbf{8}$ e58183. (doi:10.1371/journal.pone.0058183)

Fullgrabe J, Kavanagh E \& Joseph B 2011 Histone onco-modifications. Oncogene 30 3391-3403. (doi:10.1038/onc.2011.121)

Gleason DF \& Mellinger GT 1974 Prediction of prognosis for prostatic adenocarcinoma by combined histological grading and clinical staging. Journal of Urology 111 58-64.

Guccione E, Bassi C, Casadio F, Martinato F, Cesaroni M, Schuchlautz H, Luscher B \& Amati B 2007 Methylation of histone H3R2 by PRMT6 and H3K4 by an MLL complex are mutually exclusive. Nature 449 933-937. (doi:10.1038/nature06166)

Guess HA 2001 Benign prostatic hyperplasia and prostate cancer. Epidemiologic Reviews 23 152-158. (doi:10.1093/oxfordjournals. epirev.a000782)

van Haaften G, Dalgliesh GL, Davies H, Chen L, Bignell G, Greenman C, Edkins S, Hardy C, O’Meara S, Teague J et al. 2009 Somatic mutations of the histone H3K27 demethylase gene UTX in human cancer. Nature Genetics 41 521-523. (doi:10.1038/ng.349)

Hake SB, Xiao A \& Allis CD 2004 Linking the epigenetic 'language' of covalent histone modifications to cancer. British Journal of Cancer 90 761-769. (doi:10.1038/sj.bjc.6601575)

Hamamoto R, Furukawa Y, Morita M, Iimura Y, Silva FP, Li M, Yagyu R \& Nakamura Y 2004 SMYD3 encodes a histone methyltransferase 
involved in the proliferation of cancer cells. Nature Cell Biology 6 731-740. (doi:10.1038/ncb1151)

Hamamoto R, Silva FP, Tsuge M, Nishidate T, Katagiri T, Nakamura Y \& Furukawa Y 2006 Enhanced SMYD3 expression is essential for the growth of breast cancer cells. Cancer Science 97 113-118. (doi:10.1111/ j.1349-7006.2006.00146.x)

Hermanek P, Hutter RVP, Sobin LH, Wagner G \& Wittekind CF 1997 Prostate. In TNM Atlas: Illustrated guide to the TNM/pTNM classification of malignant tumors, pp 278-280. Eds P Hermanek, RVP Hutter, LH Sobin, G Wagner \& CF Wittekind. Heidelberg, Germany: Springer-Verlag.

Hirst M \& Marra MA 2009 Epigenetics and human disease. International Journal of Biochemistry \& Cell Biology 41 136-146. (doi:10.1016/ j.biocel.2008.09.011)

Jemal A, Bray F, Center MM, Ferlay J, Ward E \& Forman D 2011 Global cancer statistics. CA: A Cancer Journal for Clinicians 61 69-90. (doi:10.3322/caac.20107)

Karanikolas BD, Figueiredo ML \& Wu L 2010 Comprehensive evaluation of the role of EZH2 in the growth, invasion, and aggression of a panel of prostate cancer cell lines. Prostate 70 675-688. (doi:10.1002/ pros.21112)

Ke XS, Qu Y, Rostad K, Li WC, Lin B, Halvorsen OJ, Haukaas SA, Jonassen I, Petersen K, Goldfinger N et al. 2009 Genome-wide profiling of histone h3 lysine 4 and lysine 27 trimethylation reveals an epigenetic signature in prostate carcinogenesis. PLoS ONE 4 e4687. (doi:10.1371/journal. pone.0004687)

King CR \& Long JP 2000 Prostate biopsy grading errors: a sampling problem? International Journal of Cancer 90 326-330. (doi:10.1002/ 1097-0215(20001220)90:6 <326::AID-IJC3 > 3.0.CO;2-J)

de Kok JB, Roelofs RW, Giesendorf BA, Pennings JL, Waas ET, Feuth T, Swinkels DW \& Span PN 2005 Normalization of gene expression measurements in tumor tissues: comparison of 13 endogenous control genes. Laboratory Investigation 85 154-159. (doi:10.1038/labinvest. 3700208)

Laitinen S, Martikainen PM, Tolonen T, Isola J, Tammela TL \& Visakorpi T $2008 \mathrm{EZH} 2, \mathrm{Ki}-67$ and MCM7 are prognostic markers in prostatectomy treated patients. International Journal of Cancer 122 595-602. (doi:10.1002/ijc.23145)

Lapointe J, Li C, Higgins JP, van de Rijn M, Bair E, Montgomery K, Ferrari M, Egevad L, Rayford W, Bergerheim U et al. 2004 Gene expression profiling identifies clinically relevant subtypes of prostate cancer. PNAS 101 811-816. (doi:10.1073/pnas.0304146101)

Lee DY, Northrop JP, Kuo MH \& Stallcup MR 2006 Histone H3 lysine 9 methyltransferase G9a is a transcriptional coactivator for nuclear receptors. Journal of Biological Chemistry 281 8476-8485. (doi:10.1074/ jbc.M511093200)

Miremadi A, Oestergaard MZ, Pharoah PD \& Caldas C 2007 Cancer genetics of epigenetic genes. Human Molecular Genetics 16 R28-R49. (doi:10.1093/hmg/ddm021)

Moyer VA 2012 Screening for prostate cancer: U.S. Preventive Services Task Force recommendation statement. Annals of Internal Medicine $\mathbf{1 5 7}$ 120-134. (doi:10.7326/0003-4819-157-2-201207170-00459)

Neault M, Mallette FA, Vogel G, Michaud-Levesque J \& Richard S 2012 Ablation of PRMT6 reveals a role as a negative transcriptional regulator of the p53 tumor suppressor. Nucleic Acids Research $409513-9521$. (doi:10.1093/nar/gks764)

Popiolek M, Rider JR, Andren O, Andersson SO, Holmberg L, Adami HO \& Johansson JE 2013 Natural history of early, localized prostate cancer: a final report from three decades of follow-up. European Urology 63 428-435. (doi:10.1016/j.eururo.2012.10.002)

Ren TN, Wang JS, He YM, Xu CL, Wang SZ \& Xi T 2011 Effects of SMYD3 over-expression on cell cycle acceleration and cell proliferation in MDA-MB-231 human breast cancer cells. Medical Oncology 28 (Suppl 1) S91-S98. (doi:10.1007/s12032-010-9718-6)

Rotili D \& Mai A 2011 Targeting histone demethylases a new avenue for the fight against cancer. Genes \& Cancer 2 663-679. (doi:10.1177/ 1947601911417976)

Seligson DB, Horvath S, Shi T, Yu H, Tze S, Grunstein M \& Kurdistani SK 2005 Global histone modification patterns predict risk of prostate cancer recurrence. Nature 435 1262-1266. (doi:10.1038/nature03672)

Seligson DB, Horvath S, McBrian MA, Mah V, Yu H, Tze S, Wang Q, Chia D, Goodglick L \& Kurdistani SK 2009 Global levels of histone modifications predict prognosis in different cancers. American Journal of Pathology 174 1619-1628. (doi:10.2353/ajpath.2009.080874)

Shi Y, Lan F, Matson C, Mulligan P, Whetstine JR, Cole PA \& Casero RA 2004 Histone demethylation mediated by the nuclear amine oxidase homolog LSD1. Cell 119 941-953. (doi:10.1016/j.cell.2004.12.012)

Siegel R, Naishadham D \& Jemal A 2012 Cancer statistics, 2012. CA: A Cancer Journal for Clinicians 62 10-29. (doi:10.3322/caac.20138)

Stamey TA 1995 Making the most out of six systematic sextant biopsies. Urology 45 2-12. (doi:10.1016/S0090-4295(95)96168-2)

Van Aller GS, Reynoird N, Barbash O, Huddleston M, Liu S, Zmoos AF, McDevitt P, Sinnamon R, Le B, Mas G et al. 2012 Smyd3 regulates cancer cell phenotypes and catalyzes histone $\mathrm{H} 4$ lysine 5 methylation. Epigenetics 7 340-343. (doi:10.4161/epi.19506)

Varambally S, Dhanasekaran SM, Zhou M, Barrette TR, Kumar-Sinha C, Sanda MG, Ghosh D, Pienta KJ, Sewalt RG, Otte AP et al. 2002 The polycomb group protein EZH2 is involved in progression of prostate cancer. Nature 419 624-629. (doi:10.1038/nature01075)

Wang SZ, Luo XG, Shen J, Zou JN, Lu YH \& Xi T 2008 Knockdown of SMYD3 by RNA interference inhibits cervical carcinoma cell growth and invasion in vitro. BMB Reports 41 294-299. (doi:10.5483/BMBRep. 2008.41.4.294)

Wolters T, Vissers KJ, Bangma CH, Schroder FH \& van Leenders GJ 2010 The value of EZH2, p27(kip1), BMI-1 and MIB-1 on biopsy specimens with low-risk prostate cancer in selecting men with significant prostate cancer at prostatectomy. BJU International 106 280-286. (doi:10.1111/ j.1464-410X.2009.08998.x)

Yoshimatsu M, Toyokawa G, Hayami S, Unoki M, Tsunoda T, Field HI, Kelly JD, Neal DE, Maehara Y, Ponder BA et al. 2011 Dysregulation of PRMT1 and PRMT6. Type I arginine methyltransferases, is involved in various types of human cancers. International Journal of Cancer 128 562-573. (doi:10.1002/ijc.25366)

Zou JN, Wang SZ, Yang JS, Luo XG, Xie JH \& Xi T 2009 Knockdown of SMYD3 by RNA interference down-regulates c-Met expression and inhibits cells migration and invasion induced by HGF. Cancer Letters 280 78-85. (doi:10.1016/j.canlet.2009.02.015)

Received in final form 22 October 2013

Accepted 6 November 2013

Made available online as an Accepted Preprint

7 November 2013 http://erc.endocrinology-journals.org DOI: $10.1530 / E R C-13-0375$
(C) 2014 Society for Endocrinology Printed in Great Britain
Published by Bioscientifica Ltd 